\title{
BRIEF
}

\section{Impact of the COVID-19 Pandemic on the Emotional Intelligence of Student Pharmacist Leaders}

\author{
Kellie J. Goodlet, PharmD, Erin Raney, PharmD, Kelsey Buckley, PharmD, Titilola Afolabi, PharmD, \\ Lindsay Davis, PharmD, Rebekah M. Fettkether, PharmD, MPH, Maura Jones, PharmD, Suzanne Larson, \\ PharmD, Shawn Tennant, PharmD, MBA \\ Midwestern University, College of Pharmacy, Glendale, Arizona \\ Submitted December 18, 2020; accepted May 28, 2021; ePublished June 2021
}

\begin{abstract}
Objective. To assess changes in Emotional Intelligence Appraisal (EIA) scores following the COVID19 pandemic for pharmacy students within a voluntary cocurricular leadership development program.

Methods. Participants from the class of 2021 (pandemic group) completed an EIA self-assessment near the beginning of the leadership program in August 2019 (pre-pandemic) and at the end of the program in July 2020 (during peak first-wave COVID-19 activity) and wrote an accompanying self-reflection. To determine changes in students' emotional intelligence potentially attributable to COVID-19, differences in EIA scores from the pandemic group were compared to the pooled results of previous program cohorts (classes of 2017-2019). Prevalent themes in student self-reflections were also highlighted.

Results. Thirty-five student leaders comprised the pandemic group, with 166 students included within the control group. The proportion of students with final EIA scores indicating high emotional intelligence was greater within the pandemic group (74.3\% vs 50.6\%). While both groups had increased final EIA scores compared to baseline values, score increases were significantly higher among students in the pandemic group with respect to overall emotional intelligence and relationship management. Students commented that the pandemic highlighted the importance of emotional intelligence during stressful situations, although the lack of in-person interaction was noted as a limitation for social development.

Conclusion. Pharmacy students participating in a leadership development program during the COVID19 pandemic experienced greater increases in emotional intelligence than did the program's prepandemic cohorts. This may support the ability of health professional students to maintain resiliency through the pandemic and develop both personal and interpersonal relationship-building skills.
\end{abstract}

Keywords: emotional intelligence, leadership development, coronavirus, SARS-CoV-2, resiliency

\section{INTRODUCTION}

The COVID-19 pandemic has had a far-reaching impact on the lives of students completing professional programs in health care, including in pharmacy. Some health professionals have been energized by the pandemic, feeling proud of the opportunity to meaningfully serve patients, extend compassion and empathy, and promote public health. ${ }^{1,2}$ However, the "silent impact" of the pandemic on health care learners has been identified as an area requiring further research. ${ }^{3}$ In addition to contending with the threat to their physical health, students have had to adapt to sudden changes in curriculum structure and

Corresponding Author: Kellie J. Goodlet, PharmD, Midwestern University, College of Pharmacy, 19555 N. $59^{\text {th }}$ Ave, Glendale, AZ 85308. Tel: 623-572-3535.Email: kgoodlet@ midwestern.edu delivery, have been cut off from the day-to-day in-person comradery and support of their peers, and have often had to juggle increased domestic responsibilities, such as childcare.

Resilience and emotional regulation have been identified as characteristics associated with maintaining positive mental health and mitigating anxiety during the pandemic, including among health care professionals. ${ }^{4-6}$ Higher levels of emotional intelligence, ie, the ability to recognize, understand, and positively direct one's emotions and those of others, have been associated with decreased burnout, greater organizational commitment, and proactive implementation of positive coping strategies in response to stressful situations, including during the pandemic. ${ }^{7,8}$ The concept of emotional intelligence has been incorporated into curricular and cocurricular programming by colleges of pharmacy, as emotionally intelligent health care leaders 


\section{American Journal of Pharmaceutical Education 2022; 86 (1) Article 8519.}

are proposed to be more empathetic and engage more effectively with patients and colleagues. ${ }^{9}$ However, whether the pandemic itself has impacted students' emotional intelligence is currently unknown.

The Leadership Development Program at the Glendale campus of Midwestern University College of Pharmacy (MWU-CPG) includes a focus on enhancing the emotional intelligence of student leaders, with participants taking the Emotional Intelligence Appraisal (EIA) assessment near the beginning and end of the program. ${ }^{10}$ Participating students from the class of 2021 cohort took their initial EIA assessment prior to COVID-19 and their final assessment during a period of high first-wave coronavirus activity, providing a unique opportunity to investigate the pandemic's influence on the development of emotional intelligence. Therefore, the purpose of this study was to evaluate the impact of the COVID-19 pandemic on the EIA scores of student leaders by comparing the EIA score changes from the class of 2021 (pandemic group) to the EIA changes from prior program participants.

\section{METHODS}

The MWU-CPG Leadership Development Program was a voluntary extracurricular/cocurricular program targeting student officers that spanned six academic quarters within an accelerated, three-year Doctor of Pharmacy (PharmD) curriculum. Program activities were conducted quarterly, and consisted of large group topic discussions, readings, self-assessments, and personal reflections that were reviewed by faculty mentors. Additional details relating to program structure are outlined in a previous publication. ${ }^{10}$ Students completed an initial EIA assessment early in the program during the fall quarter, and repeated the test approximately one year after the initial assessment. An emotional intelligence construct using trait-based measures was selected to complement the program's emphasis on self-reflection and encourage students to self-evaluate how their emotional intelligence skills influence their behavior in leadership situations. Completion of the program has previously been associated with significantly increased final EIA scores. ${ }^{10}$ Students receive an overall emotional intelligence score along with scores for each emotional intelligence skill (ie, selfawareness, self-management, social awareness, and relationship management). ${ }^{11}$ These scores are established on a scale of $0-100$, derived from a normed sample, and correlated with a particular skill level: $90-100=$ "a strength to capitalize on," 80-89 = "a strength to build on," 70-79= "with a little improvement, this could be a strength," $60-69=$ "something you should work on," and 59 and below $=$ "a concern you must address." Scores of 80 and above are indicative of higher emotional intelligence, while scores less than 70 are considered indicative of lower emotional intelligence. Students self-reported their EIA pre- and post-scores to their faculty mentor, and reflected on their score changes by answering the following prompt: "Describe any changes in your EIA scores from the initial assessment and what thoughts you have about the change (if any)."

For the class of 2021 (pandemic group), students completed their initial EIA assessment in August 2019, prior to the emergence of COVID-19. Their final EIA assessment was completed in early July 2020, during peak first-wave COVID-19 activity in Arizona. ${ }^{12}$ At MWU$\mathrm{CPG}$, in-person classes were transitioned to virtual formats near the beginning of the 2020 spring quarter. The final two topic discussions within the leadership program (not directly related to emotional intelligence) were similarly transitioned to virtual formats. In order to distinguish changes in emotional intelligence potentially attributable to the COVID-19 pandemic from changes due to the leadership program or general curriculum, the EIA scores of the pandemic cohort were compared to the pooled EIA scores of participants from the classes of 2017, 2018, and 2019 (non-pandemic control group). The EIA scores of the class of 2020 were not included as they were not formally tracked; however, overall score trends were similar to the prior three classes.

The participants' scores were evaluated in aggregate within Excel. Statistical analysis consisted of paired, twotailed $t$ tests for changes in initial and final EIA scores, and unpaired, two-tailed $t$ tests for comparing the change in initial and final EIA scores between the pandemic cohort and control group. Dichotomous outcomes were compared using the chi-square or Fisher exact test. Trends in student reflection comments relating to emotional intelligence and the COVID-19 pandemic were evaluated for common themes and summarized qualitatively. This research fulfilled criteria for exempt review and was approved by the Midwestern University Institutional Review Board.

\section{RESULTS}

Thirty-five students from the class of 2021 (pandemic group) were enrolled in the MWU-CPG Leadership Development Program and submitted their initial and final EIA scores, while 166 students across three previous program cohorts comprised the non-pandemic group. In both groups, the final mean EIA scores were significantly higher than mean initial scores, including across all four emotional intelligence skills (Table 1). However, the score increase for the pandemic group was significantly higher than that of the non-pandemic group with respect to 


\section{American Journal of Pharmaceutical Education 2022; 86 (1) Article 8519.}

Table 1. Comparison of Emotional Intelligence Appraisal (EIA) Scores of Students Completing a Leadership Development Program During the COVID-19 Pandemic vs a Non-Pandemic Control Group of Students

\begin{tabular}{|c|c|c|c|c|c|}
\hline & \multicolumn{2}{|c|}{$\begin{array}{c}\text { Class of 2021, Pandemic Group } \\
(\mathrm{N}=35) \text {, Mean (SD) }\end{array}$} & \multicolumn{2}{|c|}{$\begin{array}{c}\text { Classes of 2017-2019, Control Group } \\
(\mathrm{N}=166) \text {, Mean (SD) }\end{array}$} & \multirow{2}{*}{$\begin{array}{c}\text { EIA Score Increase } \\
\text { Between the Groups, } \\
p \text { value }\end{array}$} \\
\hline & Initial & Final & Initial & Final & \\
\hline Overall & $76(8)$ & $83(6)^{a}$ & $74(8)$ & $79(9)^{\mathrm{a}}$ & .031 \\
\hline Self-Awareness & $76(10)$ & $84(9)^{\mathrm{a}}$ & $74(11)$ & $79(10)^{\mathrm{a}}$ & .075 \\
\hline Self-Management & $75(10)$ & $83(8)^{a}$ & $74(11)$ & $79(11)^{\mathrm{a}}$ & .075 \\
\hline Social Awareness & $78(11)$ & $82(9)^{a}$ & $75(11)$ & $79(11)^{\mathrm{a}}$ & .47 \\
\hline $\begin{array}{l}\text { Relationship } \\
\text { Management }\end{array}$ & $75(9)$ & $83(8)^{\mathrm{a}}$ & $74(10)$ & $78(11)^{\mathrm{a}}$ & .020 \\
\hline
\end{tabular}

${ }^{a}$ Significant statistical difference $(p<.05)$ compared to initial mean score.

overall emotional intelligence $(+7 \mathrm{vs}+5, p=.031)$ and relationship management $(+8$ vs $+4, p=.020)$. The changes in self-awareness and self-management scores were numerically higher for the pandemic group but not statistically different $(+8$ vs $+5, p=.075)$, while the change in social awareness was not different between the groups ( $+4 \mathrm{vs}+4, p=.47)$.

While both groups' initial mean scores were solidly within the "with a little improvement, this could be a strength" range, students in the pandemic group improved their mean scores to the next skill level ("a strength to build on"), while final scores from the non-pandemic group remained within the same skill level, albeit with movement toward the upper end of the range. Students in the pandemic group were also more likely to have a final overall EIA score denoting high emotional intelligence than the non-pandemic group ( $74.3 \%$ of the total class vs $50.6 \%, p=.030$ ), and were additionally less likely to have a final score suggestive of lower emotional intelligence ( $2.9 \%$ of the total class vs $18.1 \%, p=.020)$. The proportion of students at each skill level overall and for each individual emotional intelligence skill is depicted in Figure 1.

With respect to student reflection comments, six students (17.1\%) from the pandemic group explicitly ascribed changes in their EIA scores to the COVID-19 pandemic. On the positive side, students noted that having more time at home provided them with an opportunity for deeper self-reflection, resulting in greater self-awareness. The pandemic also highlighted to some students the importance of managing emotions during stressful and frustrating situations, spurring them to dedicate additional effort toward improving their emotional intelligence. One student noted a modest drop in her self-awareness score, but described the change as a positive result of her efforts to address social anxiety during quarantine and spend less time dwelling on personal stressors. Other students noted the negative effects of the pandemic on their emotional intelligence. These included limited social situations to practice emotional intelligence skills, difficulties transitioning to online learning, and feeling the need to suppress their emotions.

\section{DISCUSSION}

Among students participating in a voluntary leadership development program, greater increases in overall emotional intelligence and relationship management were observed among the class of 2021, which may reflect their experiences during the COVID-19 pandemic. Program content was not altered from prior years, suggesting that these changes occurred independently of the program's impact on emotional intelligence, or, alternatively, that the landscape of the pandemic augmented the program's impact and led the students to find the program and the concept of emotional intelligence more valuable.

The emotional intelligence skill of relationship management, which increased the most over pre-pandemic cohorts, involves taking the time to understand where others are coming from, extending grace and empathy toward others instead of rushing to judgment, and making an active effort to maintain personal relationships and show people that you care. During the transition to virtual learning, the mantra of "we are all in this together" was emphasized at MWU-CPG, as well as the need to be flexible and compassionate while navigating a new reality. Restrictions on in-person interactions may have spurred students to fortify and strengthen their relationships with friends and family, further enhancing relationship management skills. The increased need to proactively schedule virtual get-togethers to maintain relationships with classmates may have also contributed to students' ability to be "more present" during these scheduled interactions vs prior day-to-day surface-level interactions on campus.

Of note, the emotional intelligence skill of social awareness, while increased over initial mean values, did 
American Journal of Pharmaceutical Education 2022; 86 (1) Article 8519.

Overall EIA Score

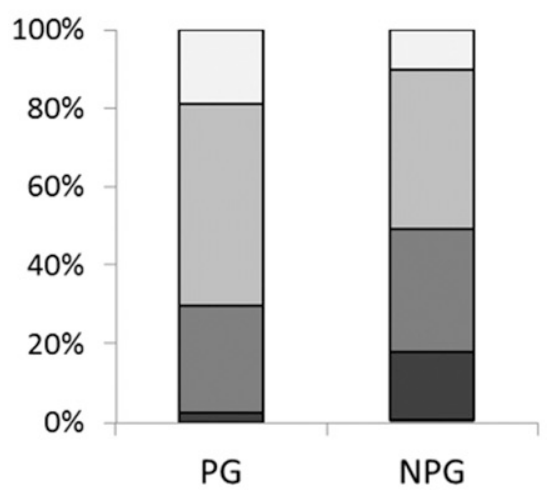

Self-Management

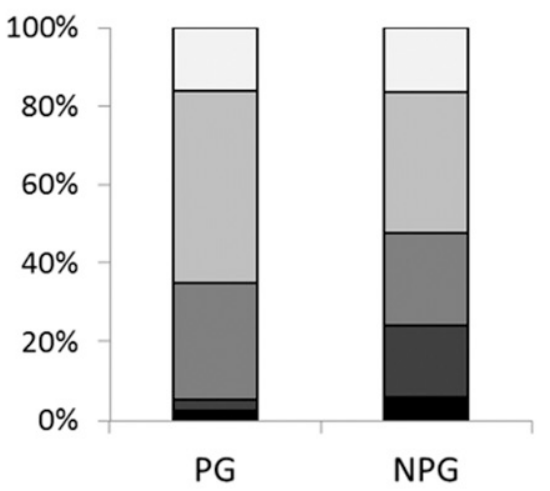

Self-Awareness

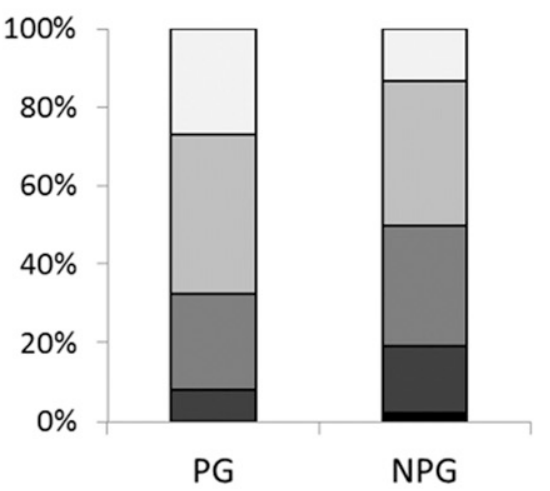

Social Awareness

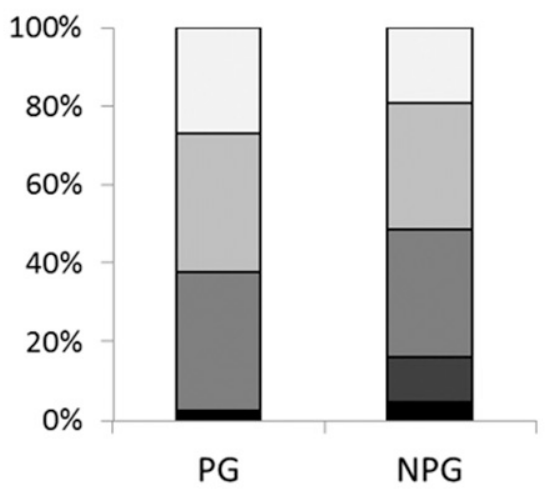

KEY:

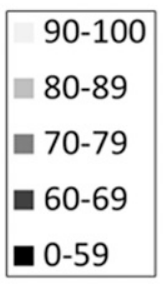

Relationship Management

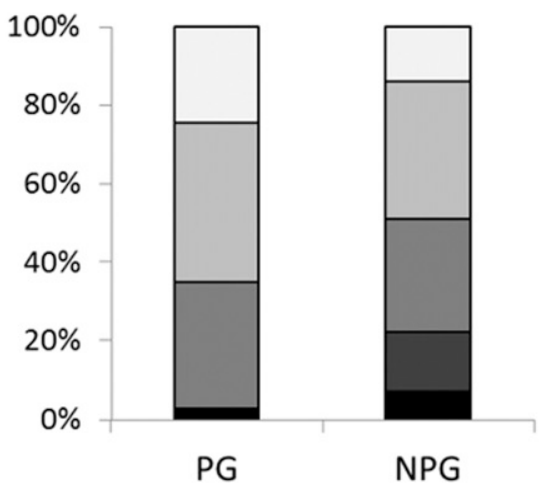

Definitions: EIA=Emotional Intelligence Appraisal; PG=pandemic group (Class of 2021), NPG=non-pandemic group (pooled results from the Classes of 2017-2019) The EIA score ranges correspond to the following skill levels: $90-100=$ "a strength to capitalize on," $80-89=" a$ strength to build on,"

70-79="with a little improvement, this could be a strength," 60-69="something you should work on," 0-59="a concern you must address."

Figure 1. Proportion of students at each emotional intelligence skill level based on their final Emotional Intelligence Appraisal score comparing the pandemic group and non-pandemic control group.

not rise more for the class of 2021 compared to the increase seen among prior cohorts. This finding is not unanticipated, given the physical distancing measures implemented to curb COVID-19 transmission. Social awareness skills may be honed by observing and listening to others more closely, and some students commented that the pandemic resulted in fewer opportunities for these interactions. It has also been recognized that people are less able to accurately perceive others' emotions (a key component of social awareness) when face masks are worn. ${ }^{13}$ Overall the results of this study are promising and suggest that the latest class of students will be equipped with the skills needed to serve as emotionally intelligent health care providers upon graduation.

Limitations of these results should be noted. Although the study design was strengthened by the inclusion of a control group, the greater increases in emotional intelligence observed in the class of 2021 may have occurred even in the absence of the pandemic. Only a minority of students related changes in their individual EIA scores to COVID-19, though they were not specifically prompted to reflect on the pandemic in their responses. Also, while most students' EIA scores increased, some saw their scores decrease, albeit a lower proportion than previous years. While lower EIA scores do not necessarily indicate decreased emotional intelligence (for example, they may represent a student becoming more self-aware), the comments from students noting the negative effect the pandemic had on their emotional health highlight that no class is homogenous. Having high emotional intelligence also does not negate the need for social support, ${ }^{14}$ and differing reactions by students to the pandemic should be anticipated. Finally, the results reflect the experiences of a single motivated group of student leaders at MWU-CPG, representing approximately onequarter of the matriculated class. Overall, these results are in line with the experiences of other health care professionals, with frontline workers and learners demonstrating an 


\section{American Journal of Pharmaceutical Education 2022; 86 (1) Article 8519.}

altruistic commitment toward helping others and extending compassion despite acknowledging personal feelings of vulnerability and anxiety. ${ }^{2,15}$

\section{CONCLUSION}

As schools and colleges of pharmacy seek to mentor students in developing leadership skills and emotional intelligence, the lessons learned from this research are noteworthy and applicable beyond this pandemic. As students navigated the uncertainties affecting multiple aspects of their professional and personal lives, the shared experience of COVID-19 may have enhanced the rapidity with which growth in their emotional intelligence, particularly relationship management skills, advanced. These findings support the theory that, with guided selfreflection and mentor support, challenges can yield opportunities for positive personal growth. Faculty can continue to encourage students to reflect on the challenges they have overcome as a catalyst for meaningful future growth.

\section{ACKNOWLEDGMENTS}

The authors would like to acknowledge Drs. Bill Bowman, Alyssa Peckham, Joie Rowles, and Tara Storjohann for their contributions in creating, maintaining, and/or delivering the described program, as well as the MWU-CPG Dean's Office for their financial and administrative support.

\section{REFERENCES}

1. Galea S. Compassion in a time of COVID-19. Lancet. 2020; 395(10241):1897-1898.

2. Zhang Y, Wei L, Li H, et al. The psychological change process of frontline nurses caring for patients with COVID-19 during its outbreak. Issues Ment Health Nurs. 2020;41(6):525-530.
3. Holt GR. The pandemic effect: raising the bar for ethics, empathy, and professional collegiality. Otolaryngol Head Neck Surg. 2020. 194599820933179.

4. Barzilay R, Moore TM, Greenberg DM, et al. Resilience, COVID-19-related stress, anxiety and depression during the pandemic in a large population enriched for healthcare providers. Transl Psychiatry. 2020;10(1):291.

5. Li JB, Yang A, Dou K, et al. Self-control moderates the association between perceived severity of coronavirus sisease 2019

(COVID-19) and mental health problems among the Chinese public. Int J Environ Res Public Health. 2020;17(13):4820.

6. Taylor R, Thomas-Gregory A, Hofmeyer A. Teaching empathy and resilience to undergraduate nursing students: a call to action in the context of covid-19. Nurse Educ Today. 2020;104524.

7. Moreno-Fernandez J, Ochoa JJ, Lopez-Aliaga I, et al. Lockdown, emotional intelligence, academic engagement and burnout in pharmacy students during the quarantine. Pharmacy (Basel). 2020;8(4):E194.

8. Prentice C, Zeidan S, Wang X. Personality, trait EI and coping with COVID 19 measures. Int J Disaster Risk Reduct. 2020;51: 101789.

9. Ward A, Hall J, Mutch J, et al. What makes pharmacists successful? An investigation of personal characteristics. J Am Pharm Assoc. 2019;59(1):23-29.e1.

10. Buckley K, Bowman B, Raney E, et al. Enhancing the emotional intelligence of student leaders within an accelerated pharmacy program. Am J Pharm Educ. 2020;84(11):8056.

11. Bradberry T, Greaves J. Emotional Intelligence 2.0. San Diego, CA: TalentSmart, Inc.; 2009.

12. Johns Hopkins University of Medicine. Impact of opening and closing decisions by state. https://coronavirus.jhu.edu/data/statetimeline/new-confirmed-cases/arizona. Accessed December 17, 2020 .

13. Carbon CC. Wearing face masks strongly confuses counterparts in reading emotions. Front Psychol. 2020;11:566886.

14. Zysberg L, Zisberg A. Days of worry: emotional intelligence and social support mediate worry in the COVID-19 pandemic. J Health Psychol. 2020;1359105320949935.

15. Gallagher TH, Schleyer AM. "We signed up for this!" - Student and trainee responses to the COVID-19 pandemic. $N$ Engl J Med. 2020;382(25):e96. 